\title{
A Chess Move Approach to 'Choices' in a Mental Cosmos
}

\author{
Jonathan C. W. Edwards ${ }^{1}$ (i)
}

Received: 17 May 2018 / Accepted: 26 March 2019 / Published online: 27 April 2019

(C) The Author(s) 2019

It is a great pleasure to respond to Henry Stapp's thoughts on fundamental issues, equally where there are points of complete agreement, and where we clearly disagree. Stapp's views are so uncompromising and so clear that they form the perfect basis for constructive dialogue.

My own view of fundamental physics is that it should, and does, follow from the basic principles identified by Leibniz (1714). Leibniz gets forgotten in the history of science because, unlike Newton, he insisted on addressing deeper questions that are not always easily approachable. And in this I see a similarity with Stapp. Both argue that there are certain awkward questions to which answers are imperative. Both lay out the a priori requirements for a solution. One may disagree with some of the conclusions they draw, but the frameworks remain robust.

The first issue is realism. Realism may have two meanings relevant here. One is that there is something dynamic really going on that we as subjects relate to: a denial of solipsism or extreme idealism. That I think we can agree on. The other is that individual instances of what is really going on can be envisaged in something like familiar terms - perhaps as waves or particles. This is where Leibniz and I would say no. However we try to describe an indivisible quantum of action it cannot be in terms of internal mechanisms envisageable in familiar terms. That would imply that the action could be divided into sub-actions and by definition, for a theory of indivisible quanta, that cannot be right.

This is not quite 'shut up and calculate', however, because there is no suggestion that, as Kant might have it, there is some unknowable thing in itself hiding from our ken. We can form a complete metaphysical account, but one that requires some abandoning of intuitive ideas concerning things like space and time. We should not be worried by that, because empirical neuropsychology tells us that the familiar appearances of space and time are merely coded signs our

Jonathan C. W. Edwards

JO.EDWARDS@UCL.AC.UK

1 University College London, London, UK brains use to tell themselves about distal events. Even Newton (1687) recognises this in his first Scholium.

An insistence on dynamic indivisibility, with Leibniz, and of course Bohr, means that 'processes 1 and 2' must somehow be made one. This leads to what I call a 'chess move' model of the quantum of action. Consider the universe as an electronic chessboard, with no material 'pieces', just a field of values. In chess, there are 64 values at any time, either null, or pawn value or queen value etc. A move has initial conditions, perhaps including knight value for Q3 and null value for QB5. A possible knight's move leads to a reversal of these values. Nothing can be, or need be, said about any sub-events in between.

What then does the wave equation describe? As I understand it, the wave equation does not describe any single actual action Ap or Aq. It may look like a description of something progressing in time but is actually a formula that gives look up tables for the probabilities of all possible actions, An, arising from a set of initial conditions. A description of an actual action defined by initial and final conditions at points in spacetime does not involve probabilities. It happened; $p=1$. As Leibniz points out, an actual action Ap entails both a beginning and an end. Without both it is not yet that unique action Ap, merely one of many possibilities with no actual identity. So there is never an electron mode Ep or photon Pp 'on its way' maybe here or maybe there. Ep is a mode whose entire unique history is actual, making it that mode.

This approach resolves the worry about supraluminal causation within a single quantum of action, as in the form of emission of two entangled photons (akin to a 'castling' move). Within a mode of action that only actually exists in toto there is no internal before or after. The spacetime metric is directionless and nothing 'progresses'. What we think of as temporal direction has nothing specifically to do with the time metric within descriptions of actions. It is the direction of sequence in which the actions connect. The bar on supraluminal influence only applies to the relations between points of connection between actions. Within an action causation is simply a matter of realisation of the possibility of that action occurring in the 
context of the total pattern of field values over what may be a vast domain of spacetime.

As Feynman (1966) points out, things get more subtle when it becomes clear that the distinction between possibility and actuality may not be black and white. There are complications here but I think Leibniz can show us how to navigate. Perhaps the secret is to see everything, as Stapp proposes, in mental terms. All that exist are actions and these, for Leibniz, are by definition also perceptions from points of view. Events are actual just to the extent that they entail relations of perception, or in dynamic terms, relations with other actions through informing influence (not unreasonably cast as 'progression in harmony'). For an action to occur, it is not necessary for any human being to perceive it directly but there must be at least one other action with which the first action connects and in that sense is 'perceived'.

What emerges from this is the idea that, rather than having a block universe, we have a universe consisting of miniblock actions, with a directionless spacetime metric, connected in sequence. Such miniblocks have been called various things including 'causal diamonds' (Savitt, 2002). I like the idea of spacetime 'sequins' joined by directional hook-and-eye connections to form the fabric of the universe. I believe this is very consistent with Stapp's own view of stepwise moves forward on an advancing quantum front.

The 'advancing' nature of this front can be challenged on the basis that if we assume all connecting points of each sequin are co-defined we get an infinite regress of actuality fixed into eternity. I think, however, that this relates to a different metaphysical question that does not affect the validity of what has been said so far and may in fact raise spurious ideas about 'passage' of time. Leibniz handles this with some skill. He points out that whatever the future is going to be it is definitely going to be that future. But that does not imply no range of possibilities for each connection. There are, in some rather counterintuitive sense, 'choices', perhaps atemporal choices, but choices all the same. The interesting question is choices for whom?

I used to consider myself an atheist. However, some years ago I realised that, perhaps surprisingly, those with a commitment to some form of cosmic mentality, including both Stapp and Leibniz, often seem to push harder on the awkward questions that underlie physical dynamics than atheist colleagues in biology and physics. I came to realise that recognition of the existence of 'Cosmic Mind' or a 'Necessary Being', rather than introducing unparsimonious duality, makes it simpler to take logical imperatives to their proper conclusions. I think that a Leibnizian concept of a 'totality of sufficient reasons' or 'necessary infinite being' cannot, and should not, be avoided. Moreover, that necessary being must, if anything does, entail mentality.

So, to be more specific, there seem to be two awkward and interconnected questions about 'choices' in world dynamics. The first is what exactly is meant by choice in this context.
The second is whether these are the choices of dynamic individuals or the choices of a universal cosmic mind.

The idea of choice being involved in fundamental (quantum) dynamics is associated with Bohr's concept of the observer's role in choosing what measurement to make. Although I may be wrong, I find it hard to relate choice in this folk-psychological sense with the emergence of a particular actual mode of action from a repertoire of possibilities. In this, it seems that I differ from Stapp. The traditional concept of choice is associated with a sense of motivation, which in turn normally implies 'reason'. Certainly, the cherished notion of free will is associated with the idea that the individual can follow their own motivations and reasons, rather than be a slave to external forces. But, as Leibniz points out, the choices associated with particular modes of action being actual lie within the spectrum of possibility that sufficient reasons leave open. Reason is that part of the story that is not optional.

As has been pointed out by others, for free will, or choice, in the popular sense, to be of any value, it should be determinate rather than stochastic. The choices associated with certain actions becoming actual, in contrast, appear to be more truly 'free' in the sense of being beyond the scope of necessity, being truly optional in a dynamic sense. This is not choice in the folk-psychological sense but it is a real actualisation of options that has to be recognised, alongside sufficient reason, to account for world dynamics.

To whom or what would this sort of choice belong? An attractive account might be that these must be the choices of the cosmic mind or necessary being since this is where the ultimate explanation for everything lies. Yet Leibniz argues differently. The necessary being provides all sufficient reasons but these choices are specifically outside reason. This gives rise to the Christian position that these dynamic choices belong to individual souls, which for Leibniz are the highest form of modes of action. What highlights the counterintuitive nature of these choices is that a soul, or mode of action, chooses its own actuality. Since it does not even exist prior to actualisation this is clearly not a choice in anything like the popular sense, but that has already been conceded. Perhaps the concept of ownership of choices of this sort is just inappropriate. I find this very hard to resolve.

An extension of this argument about the role of a necessary being or cosmic mind seems worth raising. Leibniz claims that perceiving souls are imperfect because they are finite in terms of being limited to one point of view. The necessary being is not limited in this way and is as a result infinite and, as such, perfect. That might imply that the necessary being also perceives, or knows, but from the totality of points of view. However, Leibniz also makes the point that the necessary being is not located in space and time but beyond location. Its nature is in the form of capabilities or powers. Like familiar reasons, these transcend location. The reason why coal burns is not located anywhere. Rather than attributing world dynamics to 
God's 'actions', as Malebranche did, or His perception or knowledge, Leibniz suggests talking in terms of 'understanding', again a form of power without location. This to me is an important distinction that separates what we might call cosmic mind from mind in the popular sense. I do not think we should expect cosmic mind to perceive or to know because these are inherently limited dynamic concepts anchored in location. Equally, I think it is mistake to talk of cosmic consciousness, since consciousness is a form of knowing that is again implicitly limited and located. Nevertheless, I see this as quite compatible with the notion that the origin of our reality is primarily mental, in the sense of giving rise to mental relations. The 'physical' is the way other mentality is causally reflected within each individual mental unit (or monad).

Taking fundamental dynamics in these sorts of terms my view is that Leibniz's conception of a world composed of individual dynamic units, each interacting with the whole according to the totality of sufficient reasons presages modern fundamental physics in a very comprehensive way. Moreover, he provides a rationale for why at first quantum theory appears so counterintuitive. A fundamental explanation in terms of dynamic indivisibles has to be quite unlike an account of the dynamics of material aggregates for simple logical reasons. An account of the rules of chess moves is never going to look like an account of lifting chess pieces.

It is often said that the two great remaining puzzles in science are the nature of fundamental dynamics and the nature of mind. I am in agreement with Stapp in suggesting that these two questions may be essentially one and the same. Nature is fundamentally mental. That simple analysis, however, leaves a reasonable amount of detail to fill in! My suspicion is that on the physics side the really big paradigm shifts have been completed. I personally doubt that string theory or other similar mathematical reworkings will prove crucial. Quantum field theory works remarkably well and, for a Leibnizian, is metaphysically very satisfactory! On the other hand, as a biologist, I am very aware that bringing together fundamental physics and mind within the human brain, as we should expect to be able to do, is going to need some significant paradigm shifts.

There is no space here to explore the role of indivisible dynamic modes in human perception and consciousness in detail. However, I think there is reason to be optimistic that recent condensed matter physics may provide indivisible modes of action that can make sense of qualitative experience. The observations of Bandyopadhyay's group (Sahu et al. 2013) on long-range modes in cytoskeleton elements are particularly encouraging. What I think may be more relevant to Stapp's proposals is a broader discussion of the options for the nature and role of a 'human subject' in the dynamics. If a 'choice' is to be made and made by an individual subject, what exactly is that subject, in dynamic terms? Surely, a human subject, to be efficacious, must be some form of dynamic unit, and to perceive, it must, as both Descartes and Leibniz claim, be indivisible in its relation to the world.

In terms of detailed application of basic principles, however, I part company with Leibniz (1765) here and side with his sparring partner in the New Essays on Human Understanding: John Locke. Locke held that there was no enduring human soul beyond a narrative train held together by the mechanism of memory. For Locke, there was no need to find a fundamental indivisible dynamic unit that existed throughout a human lifetime, or indeed beyond into eternity. Locke's approach is much easier to marry with a modern physics in which indivisible dynamic modes are considered evanescent. Leibniz claimed that a human soul existed for all time. As Russell said, it is hard to see how such an enduring 'action' could be indivisible, particularly when Leibniz himself appears to divide its history into a sequence of 'petite perceptions'. Whitehead seems to provide a useful alternative in making the fundamental dynamic unit a transient 'occasion of experience'. But then he wants to combine occasions into a nexus that is an enduring subject. Leibniz's denial of such combination is one of the strengths of his position.

Leibniz does of course claim a multiplicity of monadic souls (i.e. indivisible actions) within a human being. For him, each cell has its dominant mode of action, and each organ. But he insists that there is one dominant enduring rational soul. Modern neuroscience casts doubt on that. Within a human brain, it is implausible that there should be single dominant mode of action of the sort Descartes seated in the pineal. And Anscombe (1975) pointed out that we have no justification for assuming that there is a single 'I' within a brain either over time or at a point in time. Ironically, within materialist mainstream neuroscience, the prevailing view is still of a single continuous 'owner' of conscious experience, despite the denial of any unique event of dynamic integration - a position that seems less coherent than Descartes.

If we accept Locke's argument that memory can account for a sense of an enduring self when no temporally enduring indivisible unit exists, then a multiplicity of 'conscious' dynamic units both over time and at a point in time seems to be most consistent with neuroscience. The fact that each person only reports one particular type of experience at a time is consistent with there being only one pattern of external events to form the referent content of all internal experiences. Consonance is ensured by a common input from sense organs. Moreover, it is presumably merely a matter of wiring to ensure that verbal reports always reflect the content of experience for certain very specific modes of action associated with computational events within the brain at a particular level. Computers are readily programmed to report the data content of very specific sub components. Panpsychism was never the bogeyman.

This discussion only scratches the surface of unanswered questions in brain dynamics but I think issues of this sort will need resolving before we can make substantive progress in understanding our mentality. If dynamic 'choices' occur in brains, 
in association with actualisation of certain actions, as it seems they must, then these choices look to be far removed from the folk-psychological sense of the term. Observation of outside events, including those in physics experiments, will involve vast numbers of indivisible actions becoming actualised and as yet we do not have much idea which ones have the mentality we think of as that of a human subject. These events occur within a cosmic framework that we have every reason to consider 'mental' but, not being tied to location in spacetime, the source of mentality must remain ineffable.

The job in hand is to try to bring all these considerations from different disciplines together in order to get a true understanding of the relation of human subjects to their world. That interdisciplinary effort may have been relegated to low priority for much of the twentieth century. However, the motivation to address the task may now be re-emerging, in good part thanks to individuals like Henry Stapp never taking their eye off the ball.

\section{Compliance with Ethical Standards}

Conflict of Interest The author declares that he has no conflicts of interest.

Open Access This article is distributed under the terms of the Creative Commons Attribution 4.0 International License (http:// creativecommons.org/licenses/by/4.0/), which permits unrestricted use, distribution, and reproduction in any medium, provided you give appropriate credit to the original author(s) and the source, provide a link to the Creative Commons license, and indicate if changes were made.

\section{References}

Anscombe, G. E. M. (1975). The first person. In Guttenplan SD (Ed.), Mind and language. Oxford, OUP. https://ifac.univ-nantes.fr/IMG/ pdf/Anscombe-The_First_Person.pdf

Feynman, R. (1966). Lectures on physics.volume 3. http://bayanbox.ir/ view/8431816323796678904/Feynman-lecthre-vol3-quantum.pdf

Leibniz, G. W. (1714). Monadology. http://www.earlymoderntexts.com/ assets/pdfs/leibniz1714b.pdf

Leibniz, G. W. (1765). New essays on human understanding. http://www. earlymoderntexts.com/assets/pdfs/leibniz1705book1.pdf

Newton, I. (1687). Philosophiae Naturalis Principla Mathematica http:// sites.trin.cam.ac.uk/manuscripts/NQ_16_200/manuscript.php? fullpage $=1 /$

Sahu, S., Ghosh, S., Ghosh, B., \& Bandyopadhyay, A. (2013). Atomic water channel controlling remarkable properties of a single brain microtubule: correlating single protein to its supramolecular assembly. Biosensors and Bioelectronics, 47C, 141-148.

Savitt, S. (2002). On absolute becoming and the myth of passage. In C. Callender (Ed.), Time, reality \& experience. Cambridge: CUP.

Publisher's Note Springer Nature remains neutral with regard to jurisdictional claims in published maps and institutional affiliations. 